\title{
The optimization research of MHD propulsion pipeline of the ship based on the MHD model
}

\author{
Chong Meng ${ }^{1, a}$ \\ ${ }^{1}$ North China Electric Power University (Baoding). Huadian Road No. 689. Baoding City in \\ Hebei ,China \\ awamengchong@163.com
}

Keywords: MHD propulsion, numerical simulation, goal programming model, feedback net work.

\begin{abstract}
Based on the MHD model of FLUENT software, we do a two dimensional numerical simulation about the MHD propulsion pipeline of the ship in electromagnetic coupling field. Having validated the established results, according to the research object, we make an appropriate simplification and assumption for propeller pipeline model, then establish the optimization model aiming at the propeller working efficiency. By comparing the working efficiency of several typical lines, it is concluded that under the same external condition, the inflexed pipeline has more efficient performance. Next, we do an optimization research about the specific parameters of quadratic curve which is comparatively common in the inflexed pipeline. And through establishing the feedback network based on numerical calculation, we discuss the methods of solving the optimization model.
\end{abstract}

\section{Introduction}

The MHD propulsion system was proposed in the 1870s, and in the 80s many people such as George Geelong in the University of Kobe, Japan applied the superconducting magnet to the MHD propulsion, and developed the corresponding settings[1].The research emphasis of the former Soviet Union was distinguished from Japan. The former Soviet Union mainly focused on the aspects of improving the intensity of magnetic field, and put forward the superconducting magnet with the structure of spiral pipeline.In the 1890s, China began to do the related researches and reached to a higher level. However, currently the optimization studies on the magnetic fluid are less. Therefore, we independently set up a mathematical model in our study and discuss its solving methods.

\section{Mathematical model}

The theoretical analysis model consists of the dynamics equations of incompressible fluid including the electromagnetic force and the electromagnetic field equations[2].

\subsection{The dynamics equations of incompressible fluid.}

The continuity equation: $\Delta \cdot \vec{U}=0$;

The momentum equation: $\rho \frac{d \vec{U}}{d t}=-\Delta P+\mu \Delta^{2}+\vec{j} \times \vec{B}$;

The Energy equation $\rho \frac{d}{d t}\left[U^{2} / 2+e+P / \rho\right]=f \times \mathrm{U}-\mathrm{q}$

Among them: $U$ :flow speed, $\rho$ : current density, $P$ :pressure; $\mu$ :fluid viscosity; $f$ : volume force(electromagnetic force)[3].

\subsection{The electromagnetic field equations.}

electromagnetic force: $\vec{f}=\vec{j} \times \vec{B}$ 


\section{Numerical simulation and analysis}

\subsection{Calculation model.}

The simulation calculating the range of flow field is a MHD propulsion channel. The flo $\mathrm{w}$ entry consists in yoz plane,and the flow direction is $\mathrm{x}$ positive direction. The outermost $\mathrm{l}$ ayer is the solid wall, and the entry center layer is the magnetic liquid. The size of magnet ic field along the z-axis negative direction is $1 \mathrm{~T}$ uniform magnetic field. And electric field i $\mathrm{s}$ along the $\mathrm{y}$-axis negative direction. What flows in the channel is the incompressible con ducting fluid. The viscosity, the electrical conductivity[4].

We adopt the pressure solver, the explicit or uncoupled solving format in calculating, and load the energy equation. The flow condition of MHD is turbulence, due to its high flow velocity. The $\mathrm{k}-\mathcal{E}$ equation turbulence model is adapted in turbulence model. The SIMPLE a lgorithm is used in pressure-velocity coupling. The first-order upwind is applied in flow equ ation, magnetic induction equation, turbulent kinetic energy equation and energy equation.

3.2 The simulation results and comparative analysis of computational fluid dynamics.

We consider the channel inlet velocity as a calculation condition. When the applied mag netic field $\mathrm{B}_{0}=1 \mathrm{~T}$, the simulation result of fluid speed in the channel is shown in fig.1, and the pressure diagram is shown in fig. 2 .

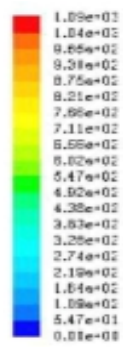

Fig. 1 the velocity profile in the channel
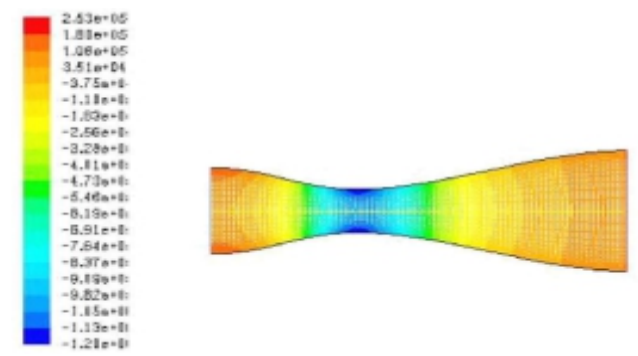

Fig. 2 the pressure profile in the channel

From the Velocity profile, we can see the fluid velocity in the propeller channel firstly increase, then decreases. There is no difference between the velocity profile and the ordinary fluid.

From the Pressure profile, we know the left side of nozzle is a high pressure area and $t$ he right side is a low pressure area. Under the effect of pressuredifference on the both end $\mathrm{s}$, gas begin to flow to the lower pressure area. Gasvelocity is fastest in the channel throat, and its pressure is minimal.

\subsection{The speed comparison under different magnetic field.}

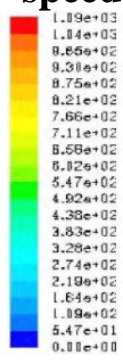

Fig.3 the velocity profile of $\mathrm{B}=1.0 \mathrm{~T}$

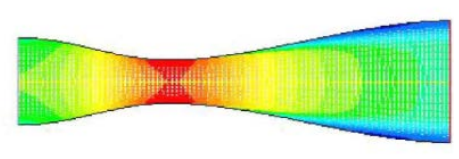

From the two different profiles, when the magnetic field intensity is $1.0 \mathrm{~T}$, the maximum velocity in the channel is $1094.426 \mathrm{~m}$ per second, but when the intensity is $1.5 \mathrm{~T}$, the maximum velocity is $775.630 \mathrm{~m}$ per second. This suggests that the fluid is blocked under the influence of electromagnetic force. And the stronger the magnetic field intensity is, the greater the blocking effects to plasma is.

\section{The establishment and analysis of optimization model}

4.1 The output power of MHD propeller.

$$
\frac{d F}{d t}=\int_{x} A_{x} \bar{v}_{x} \frac{\rho B U}{e R} d x=\frac{\rho B U}{e R} \int_{0}^{L} A_{x} \bar{v}_{x} d x
$$




$$
F=\int_{x} A_{x} \bar{v}_{x} \frac{\rho B U}{e R} d x=\frac{\rho B U}{e R} \int_{0}^{T} \int_{0}^{L} A_{x} \bar{v}_{x} d x d t
$$

4.2 The input power of MHD propeller.

$$
P_{i n}=\frac{U^{2}}{R} \quad R=\frac{b}{\delta A}
$$

4.3 The goal of optimization-the working efficiency of MHD propeller.

$$
\eta=\frac{P_{\text {out }}}{P_{\text {in }}}=\frac{L \rho B}{e U T} \int_{0}^{T} \int_{0}^{L} A_{x} \bar{v}_{x} d x d t
$$

\subsection{The comparison of several pipes output efficiency.}

First, considering the two kinds of pipelines, straight pipe and line pipe which consists of the outer fold and the inflexed.

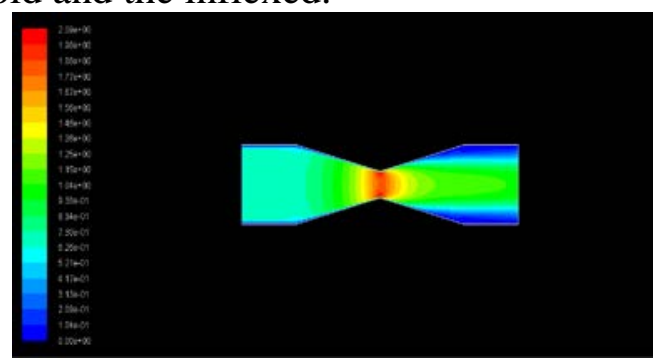

Fig.5 The velocity field in inflexed pipe

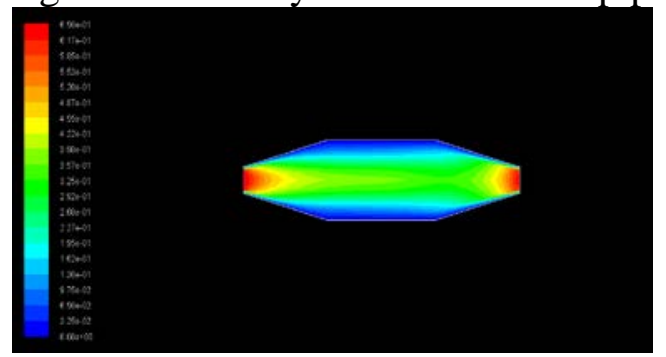

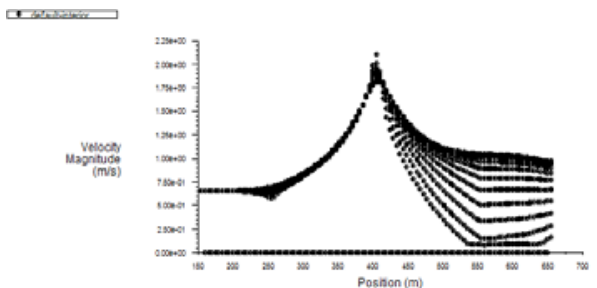

mentaven

Fig.6 The velocity curve of cross sections
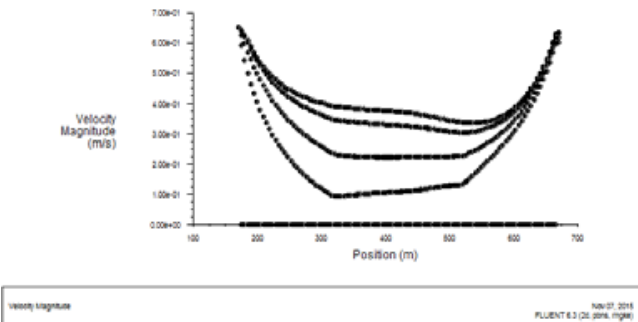

Fig.7 The velocity field in outside fold pipe Fig.8 The velocity curve of cross sections

From the velocity curves of three pipelines, it is easy to draw a conclusion that the outer fold pipeline is the last one to use. Because it can produce the maximum velocity of Lorentz force appeared at the entrance of pipeline. And its maximum velocity is merely the minimum velocity of mainstream area of inflexed pipeline. Under the same external velocity, its output power is lowest, and the efficiency is worst.We compare the output power of straight pipeline and inflexed pipeline by establishing model quantities.Because discretization can use summation instead of integral, the output power of inflexed pipe is 1061.7 $\frac{L \rho B U T}{e R T}$.the output power of straight pipe is $858 \frac{L \rho B U T}{e R T}$.

Therefore, it is a better choice to use inflexed pipeline under the same external condition.

\section{Conclusion}

We analysis the velocity and pressure field of magnetic fluid movement within the MHD propulsion pipeline by using FLUENT numerical simulation, and establish the programming model aiming at operating efficiency. Next, we compare the shape and dimension efficiency of MHD propulsion pipeline, then discuss the solving methods of model and put forward suggestions about the optimization design of pipe size in given conditions.

\section{References}

[1]Hou Helan, Zhang Shiwei, Yang Naiheng, Li Yunqi.The Development And Application of Magnetic Technology[J],Vacuum,1999,05:8-12. 
[2]Friauf J B,Electromagnetic Ship Propulsion Journal of Amer.Soc.Of Naval Engineers,139-142, 1961.

[3]ALLen Gardner B, Michael S S. AirfoiL design using a genetic algorithm and an inverse method[R].

[4]Qiulei Ding,Xiangpei Hu,Lijun Sun,Yunzeng Wang. An improved ant colony optimization and its application to vehicle routing problem with time windows[J]. Neurocomputing,2012 